\title{
Atuação fisioterapêutica e qualidade de vida de pacientes com Traumatismo Raquimedular: uma revisão integrativa
}

\author{
Physiotherapeutic performance and quality \\ of life of patients with spinal cord injury: an \\ integrative review
}

\author{
Francisco Valter Miranda Silva ${ }^{1}$ (1) \\ Antonia Nathiara Janot Silva² (1) \\ Dágila Mayara Pimenta de Castro ${ }^{3}$ (1)
}

Raquel Pinto Ribeiro4 (1)

Thais de Oliveira Sales ${ }^{5}$ (1)

Paula Pessoa de Brito Nunes $^{6}$ (1)

'Autor para correspondência. Centro Universitário Ateneu (Fortaleza). Ceará, Brasil. valtermiranda15@gmail.com
${ }^{2 \cdot 6}$ Centro Universitário Ateneu (Fortaleza).Ceará, Brasil. nathiarajr@gmail.com, dagila_mayara@hotmail.com, raquel4114ribeiro@gmail.com,
thaisales95@gmail.com, paula.nunes@uniateneu.edu.br

RESUMO | INTRODUÇÃO: O traumatismo Raquimedular (TRM) é caracterizado por uma lesão na medula espinal, que gera alterações nas funções motoras e sensitivas. Diante destas alterações que comprometem as condições de saúde dos indivíduos acometidos, a fisioterapia atua na reabilitação desses pacientes. OBJETIVO: Revisar a literatura científica e analisar a atuação fisioterapêutica e a qualidade de vida dos pacientes acometidos por TRM. MÉTODOS: Trata-se de uma revisão integrativa da literatura com a busca de artigos nas bases de dados: Scientific Electronic Library Online (SciELO), Biblioteca Virtual em Saúde (BVS), Literatura Latino-americana e do Caribe (LILACS) e PubMed, com a utilização do cruzamento de três descritores em inglês e português indexados respectivamente no Medical Subject Headings (MeSH) e Descritores em Ciências da Saúde (DeCS). Foram inclusos estudos publicados no período de janeiro de 2015 a maio de 2020. RESULTADOS: A Busca resultou em 8 artigos elegíveis para compor o escopo desta revisão. Verificou-se que a fisioterapia atua com diversas abordagens, tais como exercícios aeróbicos, uso da realidade virtual, eletroestimulação entre outros. As condutas encontradas nos estudos apresentaram efeitos benéficos que repercutem na qualidade de vida de indivíduos acometidos por TRM. CONCLUSÕES: Foi verificado que diversas abordagens fisioterapêuticas podem contribuir para a melhor qualidade de vida dos pacientes com TRM, sendo de suma importância o acompanhamento profissional especializado para o bem-estar físico e psicológico desses pacientes.

PALAVRAS-CHAVE: Fisioterapia. Qualidade de vida. Traumatismos da Medula Espinal.
ABSTRACT | INTRODUCTION: Spinal cord trauma (MRT) is altered by a spinal cord injury, which generates changes in motor and sensory functions. In view of these changes that are compromised as the health conditions of patients, physical therapy acts in the rehabilitation of these patients. OBJECTIVE: To review the scientific literature and analyze physical activity in the quality of life of patients affected by SCI. METHODS: This is an integrative literature review with the search for articles in the databases: Scientific Electronic Library Online (SciELO), Virtual Health Library (VHL), Latin American and Caribbean Literature (LILACS) and PubMed, with the use of the crossing of three descriptors in English and Portuguese indexed respectively in Medical Subject Headings (MeSH) and Health Sciences Descriptors (DeCS). Studies published from January 2015 to May 2020 were included. RESULTS: The search resulted in 8 qualified articles to compose the scope of this review. It was found that physiotherapy works with several approaches, such as aerobic exercises, use of virtual reality (VR), electrostimulation, among others. The conducts found in beneficial studies beneficial in the quality of life of individuals affected by TRM. CONCLUSIONS: It was found that several physiotherapeutic approaches can contribute to a better quality of life for patients with $\mathrm{SCl}$, with specialized professional monitoring being extremely important for the physical and psychological well-being of these patients.

KEYWORDS: Physical Therapy Specialty. Quality of life. Spinal Cord Injuries. 


\section{Introdução}

O traumatismo Raquimedular (TRM) é caracterizado por uma lesão que ocorre na medula espinal, que gera alterações nas funções motoras e sensitivas, comprometendo o sistema nervoso simpático e parassimpático ${ }^{1}$. A Lesão medular (LM) classifica-se em parcial ou total, com déficits temporários ou permanentes, onde a gravidade da patologia tem relação com o nível e local da lesão?

No mundo, estima-se que a incidência de TRM está entre 2,23 e 7,55 casos a cada 10 mil habitantes. No Brasil, a incidência estimada é de $130 \mathrm{mil}$, tendo os acidentes automobilísticos como principal causa de LM, além quedas e ferimentos por arma de fogo. Os indivíduos mais acometidos são sexo masculino, com idade média de 33 anos, onde tais dados associamse a fatores como desrespeitos as leis de transito e a violência urbana ${ }^{1}$.

Os indivíduos com TRM apresentam espasticidades, alterações sensoriais, perda de força muscular e equilíbrio, entre outros agravos, onde o nível de LM e sua localização estabelece o grau de comprometimento e extensão da lesão, podendo ocasionar diversas disfunções como o declínio funcional, que interfere negativamente na realização de Atividades de Vida Diária (AVDs), e consequentemente em sua Qualidade de Vida (QV) 3 .

De acordo com a Organização mundial de Saúde (OMS), a QV refere-se a tudo que engloba desde a saúde mental, física e psicológica, até todo o contexto social e cultural em que esse indivíduo vive e que está exposto. Portanto, as alterações causas pelo TRM, geram nos pacientes acometidos, dependência funcional e quadros depressivos que influenciam diretamente na QV4.

Diante disso, para que esses pacientes tenham uma melhor QV após o acometimento desta patologia, é preciso um programa de reabilitação com abordagem multidisciplinar ${ }^{5}$. Incluso neste programa, a fisioterapia é de suma importância na reabilitação funcional desses indivíduos, podendo assisti-los tanto na fase imediata quanto tardia, com finalidade de recuperar a funcionalidade e independência, pois utiliza-se diversas abordagem de acordo com o quadro clínico do paciente ${ }^{6}$.
No entanto, na maioria dos casos de TRM o processo de reabilitação é demorado, onde o afastamento as atividades laborais e gastos com o tratamento, envolvem questões sociodemográficas e configura um problema de saúde pública, devido a prevalência da doença ocorrer em indivíduos economicamente ativos. Portanto, a reabilitação após o acometimento da lesão pode interferir de forma direta na QV.

Ademais, estando a fisioterapia dentro do processo de reabilitação do TRM, esta pesquisa teve como objetivo revisar a literatura científica e analisar a atuação fisioterapêutica e a qualidade de vida dos pacientes acometidos por TRM.

\section{Metodologia}

Realizou-se uma revisão integrativa de literatura. A busca ocorreu no período de março a maio de 2020, nos portais/bases de dados eletrônicos Biblioteca Virtual em Saúde (BVS), Scientific Electronic Library Online (SciELO), Literatura Latino-americana e do Caribe em Ciências da Saúde (LILACS) e PubMed, com um levantamento e análise crítica da literatura sobre a atuação fisioterapêutica na qualidade de vida de pacientes com TRM.

Utilizou-se o cruzamento de três descritores indexados respectivamente em português no Descritores em Ciências da Saúde (DeCS) e em inglês no Medical Subject Headings (MeSH), associados ao operador lógico booleano $A N D$, formando os seguintes grupos de palavras: Traumatismos da medula espinal $A N D$ Qualidade de vida AND Fisioterapia e Epinal cord injuries AND Quality of life AND Physical therapy.

Foram inclusos artigos observacionais e/ou de intervenção, que abordassem a atuação fisioterapeuta e a QV em TRM, disponíveis em texto completo nos idiomas inglês, português e espanhol, publicados no período de janeiro de 2015 a maio de 2020. Como critérios de exclusão adotou-se, produções do tipo anais, documentos técnicos, editoriais, resenhas, comentários, artigos de reflexão, teses, dissertações e aqueles não correspondentes com a questão do estudo, bem como duplicidade de artigos. 
Após o cruzamento dos descritores, a busca resultou em 204. Diante dessa primeira seleção amostral foi realizada a leitura criteriosa dos títulos e resumos dos artigos selecionados por dois pesquisadores independentes. Logo após a seleção das publicações por essa filtragem, elas continuaram sendo examinadas pelos dois pesquisadores e diante de qualquer desacordo entre esses, o mesmo foi resolvido por um terceiro. Após essa análise dos títulos e resumos, foram selecionados 8 artigos para serem lidos na íntegra. Para avaliação da qualidade metodológica dos Ensaios Clínicos Randomizados (ECR), utilizou-se a escala Physiotherapy Evidence Database (PEDro), sendo aplicada nos estudos encontrados e pontuado de forma independente pelos referidos pesquisadores, no caso discordâncias, estas foram resolvidas por um terceiro investigador.

\section{Resultados}

Foram encontrados 204 artigos nas bases de dados. Deste total, após a utilização dos critérios de inclusão e exclusão, foram selecionados 33 artigos, distribuídos nas bases de dados: PubMed (8), SciELO (9), LILACS (8) e BVS (8), sendo os duplicados desconsiderados $(n=1)$, dos 33 artigos após a leitura dos títulos e resumos foram excluídos 21 por não responderem ao objetivo desta pesquisa. Assim, foram selecionados 8 artigos para leitura na integra, sendo estes inclusos nesta revisão conforme demonstrado na figura 1.

Figura 1. Descrição da seleção, avaliação e inclusão dos estudos que abordam a fisioterapia e a qualidade de vida de pacientes com TRM. Fortaleza - CE, Brasil, 2020

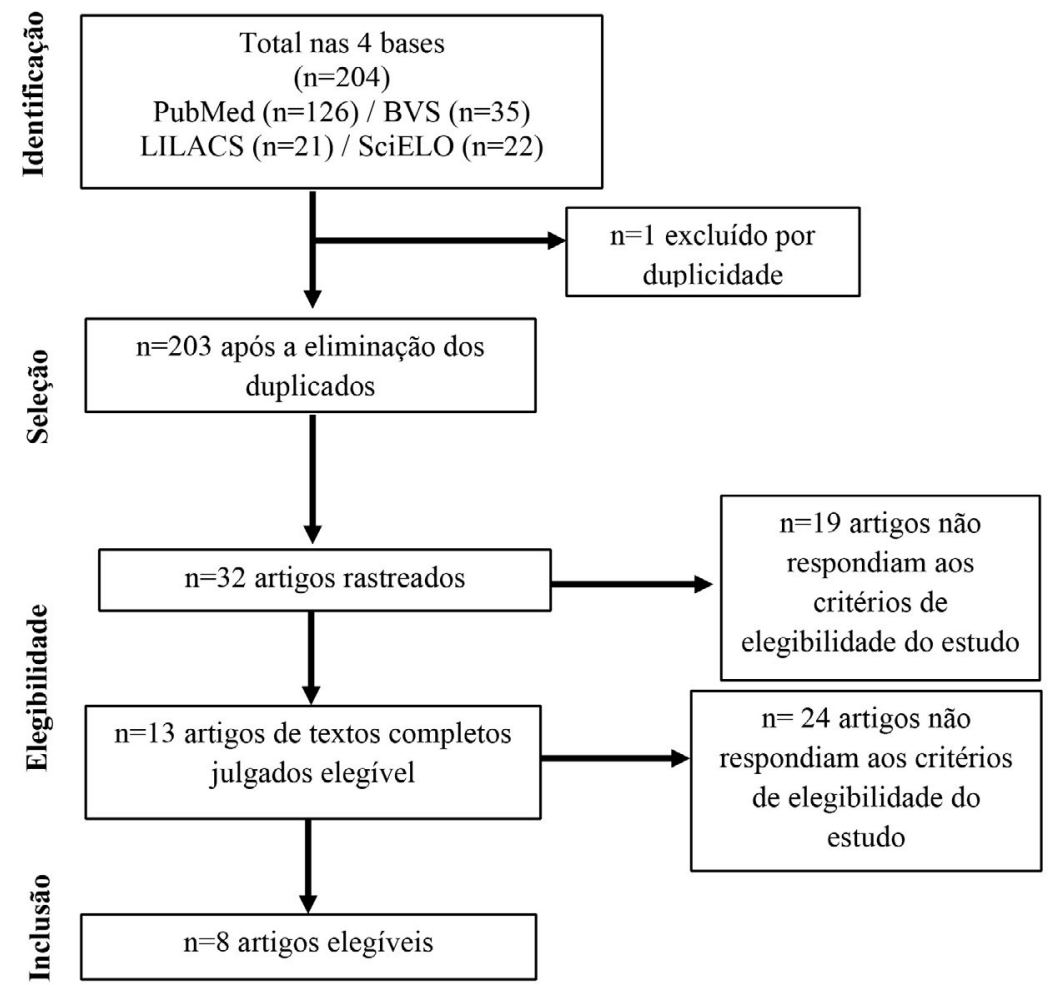

Fonte: Os autores (2020). 


\section{Descrição dos estudos}

A organização e tabulação dos dados extraídos dos 8 artigos estão descritos nas tabelas 1 e 2 de acordo com as seguintes características: autores, ano de publicação, país, população de estudo e delineamento (Tabela 1) e objetivos, instrumentos de avaliação e os principais achados (Tabela 2).

De acordo com a tabela 1, quanto ao ano de publicação, existem estudos presentes entre os anos de 2016 a 2019. Os estudos em sua maioria foram desenvolvidos no Brasil e os demais em países do continente Europeu e Norte Americano. A população com maior prevalência nos estudos são cadeirantes com diferentes faixas etárias. Em relação ao delineamento, quatro estudos são de intervenção e os demais são do tipo transversal.

Tabela 1. Caracterização dos artigos que abordam a fisioterapia e a qualidade de vida de pacientes com TRM. Fortaleza/CE, Brasil, 2020

\begin{tabular}{|c|c|c|c|c|}
\hline Autor & Ano & País & $\begin{array}{l}\text { População de estudo } \\
\text { (n) }\end{array}$ & Delineamento da Pesquisa \\
\hline FENIMA N et al. ${ }^{9}$ & 2016 & Brasil & 72 Pacientes (40 anos) & Estudo transversal \\
\hline MOTA; RIBEIRO. ${ }^{10}$ & 2016 & Brasil & 118 Pacientes (37,4 anos) & Estudo transversal \\
\hline RICHARDSON et al. ${ }^{11}$ & 2016 & EUA & 69 Pacientes (42 anos) & Estudo transversal \\
\hline AKKURT et $a l^{12}$ & 2017 & Turquia & $\begin{array}{c}40 \text { Pacientes (15 a } 65 \\
\text { anos }\end{array}$ & $\begin{array}{l}\text { Ensaio clínico controlado } \\
\text { randomizado }\end{array}$ \\
\hline LEÃO et $a l^{13}$ & 2017 & Brasil & $\begin{array}{c}15 \text { Pacientes (22 a } 50 \\
\text { anos) }\end{array}$ & Estudo de Caso \\
\hline MARESCA et $a l^{14}$ & 2018 & Itália & 1 Paciente & Estudo de Caso \\
\hline $\begin{array}{c}\text { SIVARAMAKRISHNAN et } \\
\text { al. }{ }^{15}\end{array}$ & 2018 & Noruega & 22 Pacientes (39 anos) & Duplo-cego randomizado \\
\hline CALLIGA et al. ${ }^{16}$ & 2019 & Brasil & $\begin{array}{c}38 \text { Pacientes (18 a } 65 \\
\text { anos) }\end{array}$ & Estudo transversal \\
\hline
\end{tabular}

Fonte: Dados da pesquisa (2020). 
Tabela 2. Apresentação dos artigos de acordo com os objetivos e os principais achados em relação a fisioterapia e a qualidade de vida de pacientes com TRM. Fortaleza/CE, Brasil, 2020

\begin{tabular}{|c|c|c|c|}
\hline Autor/ Ano & Objetivo do estudo & $\begin{array}{c}\text { Instrumentos de } \\
\text { avaliação }\end{array}$ & Principais achados \\
\hline $\begin{array}{l}\text { FENIMA N et al., } 2016 . \\
9\end{array}$ & $\begin{array}{l}\text { Criar e testar as } \\
\text { propriedades } \\
\text { psicométricas de um } \\
\text { instrumento específico } \\
\text { para quantificação da } \\
\text { QV em LM } \\
\end{array}$ & $\begin{array}{l}\text { Qualidade de vida } \\
\text { na lesão medular } \\
1 \text { (QVLM1). }\end{array}$ & $\begin{array}{l}\text { O Questionário de Avaliação da QV de } \\
\text { Indivíduos com LM foi criado com } \\
\text { metodologia adequada e a avaliação das } \\
\text { propriedades psicométricas traduzem em } \\
\text { um instrumento válido, confiável, } \\
\text { consistente e sensível às mudanças. }\end{array}$ \\
\hline $\begin{array}{l}\text { MOTA; RIBEIRO., } \\
2016^{10}\end{array}$ & $\begin{array}{l}\text { Identificar a prevalência } \\
\text { de úlceras por pressão } \\
\text { em portadores de TRM } \\
\text { e analisar sua QV. }\end{array}$ & WHOQOL- bref & $\begin{array}{l}\text { Observou-se uma elevada prevalência de } \\
\text { úlceras por pressão entre pessoas com } \\
\text { TRM. A avaliação da QV elucida uma } \\
\text { insatisfação significativa dos participantes, } \\
\text { principalmente quanto aos aspectos } \\
\text { físicos. }\end{array}$ \\
\hline $\begin{array}{l}\text { RICHARDSON et al., } \\
2016^{11}\end{array}$ & $\begin{array}{l}\text { Avaliar o efeito das } \\
\text { mudanças na dor na } \\
\text { mobilidade e } \\
\text { independência física e } \\
\text { QV em pessoas com } \\
\text { TRM. }\end{array}$ & $\begin{array}{l}\text { Short Form Health } \\
-12\end{array}$ & $\begin{array}{l}\text { Interferência da dor nociceptiva basal é } \\
\text { maior satisfação com a QV relacionada à } \\
\text { vida e à saúde mental. A interferência da } \\
\text { dor neuropática foi associada à mudança } \\
\text { na independência física. }\end{array}$ \\
\hline AKKURT et al., $2017^{12}$ & $\begin{array}{l}\text { Investigar os efeitos do } \\
\text { exercício aeróbico em } \\
\text { Pacientes com TRM. }\end{array}$ & Intervenção & $\begin{array}{l}\text { O exercício aeróbico de membro inferior } \\
\text { realizado por pacientes com lesão } \\
\text { medular, melhora suas capacidades de } \\
\text { exercícios aeróbicos, melhorando os } \\
\text { aspectos físicos da QV. }\end{array}$ \\
\hline LEÃO et al., $2017^{13}$ & $\begin{array}{l}\text { Verificar a influência da } \\
\text { realidade virtual (RV) } \\
\text { sobre o equilíbrio de } \\
\text { tronco e QV de } \\
\text { indivíduos com TRM. }\end{array}$ & Intervenção & $\begin{array}{l}\text { O protocolo de tratamento utilizado para } \\
\text { o estudo se mostrou eficaz para o } \\
\text { equilíbrio e QV, otimizando sua } \\
\text { funcionalidade e proporcionando uma } \\
\text { melhor QV. }\end{array}$ \\
\hline MARESCA et al., $2018^{14}$ & $\begin{array}{l}\text { Avaliar os resultados } \\
\text { cognitivos e motores } \\
\text { após um treinamento } \\
\text { de reabilitação } \\
\text { combinado usando } \\
\text { uma abordagem } \\
\text { cognitiva padrão e RV. }\end{array}$ & Intervenção & $\begin{array}{l}\text { A RV combinada de treinamento cognitivo } \\
\text { padrão pode ser eficaz em melhorar a } \\
\text { recuperação motora e cognitiva em } \\
\text { pacientes com TRM, além da redução } \\
\text { notável da ansiedade e sintomas } \\
\text { depressivos, que melhora o aspecto } \\
\text { emocional e consequentemente a QV. }\end{array}$ \\
\hline $\begin{array}{l}\text { SIVARAMAKRISHNAN } \\
\text { et al., } 2018^{15}\end{array}$ & $\begin{array}{l}\text { Comparar os efeitos da } \\
\text { estimulação elétrica } \\
\text { nervosa transcutânea e } \\
\text { da função elétrica } \\
\text { estimulação na } \\
\text { espasticidade de } \\
\text { membros inferiores em } \\
\text { pacientes com TRM. }\end{array}$ & Intervenção & $\begin{array}{l}\text { Através da estimulação elétrica nervosa } \\
\text { transcutânea (TENS) e da estimulação } \\
\text { elétrica funcional (FES) têm potencial para } \\
\text { ser usado como adjuvante terapêutico } \\
\text { para aliviar a espasticidade, } \\
\text { proporcionando assim, maior ganho de } \\
\text { funcionalidade que aumenta a QV. }\end{array}$ \\
\hline CALLIGA et al., $2019^{16}$ & $\begin{array}{l}\text { Investigar os fatores } \\
\text { que interferem no } \\
\text { retorno ao trabalho em } \\
\text { indivíduos com TRM. }\end{array}$ & $\begin{array}{l}\text { Avaliação do } \\
\text { prontuário. }\end{array}$ & $\begin{array}{l}\text { O retorno ao trabalho mostra- se, } \\
\text { relevante para o adulto com paraplegia } \\
\text { traumática, na medida em que } \\
\text { evidenciamos melhor QV, maiores } \\
\text { recebimentos e maior poder de compra } \\
\text { entre os lesionados que estavam } \\
\text { trabalhando. }\end{array}$ \\
\hline
\end{tabular}


Tabela 3. Qualidade metodológica de acordo com a escala PEDro. Fortaleza/CE, Brasil, 2020

\begin{tabular}{|c|c|c|c|c|c|c|c|c|c|c|c|c|}
\hline \multirow{2}{*}{ Autor/Ano } & \multicolumn{11}{|c|}{ Critérios* } & \multirow{2}{*}{$\begin{array}{c}\text { Pontuação } \\
\text { Total }\end{array}$} \\
\hline & 1 & 2 & 3 & 4 & 5 & 6 & 7 & 8 & 9 & 10 & 11 & \\
\hline AKKURT et al. ${ }^{12}$ & - & 1 & - & 1 & - & - & 1 & - & - & 1 & 1 & 5 \\
\hline $\begin{array}{l}\text { SIVARAMAKRISHNAN et al., } \\
2018 .{ }^{15}\end{array}$ & - & 1 & - & 1 & - & - & 1 & 1 & - & 1 & 1 & 6 \\
\hline
\end{tabular}

Fonte: Dados da pesquisa (2020).

\section{Discussão}

O estudo buscou verificar atuação fisioterapêutica e a QV de pacientes com TRM. Sendo uma patologia traumática na medula espinhal, o TRM torna o indivíduo dependente devido às limitações causadas pela lesão como: alterações sensoriais e motoras, disfunções sexuais, menor convívio social, incapacidade de locomoção e realização de atividades laborais e AVD's, gerando assim uma baixa na qualidade de vida.

Akkurt et al. ${ }^{12}$ com a maioria da amostra composta pelo sexo masculino, verificou a importância da prática de atividade física realizada por uma equipe multiprofissional, na prevenção de doenças secundárias e comorbidades como doenças cardiovasculares, diabetes, hipertensão dentre outras. Resultados como o de Almeida et al. ${ }^{17}$ e kawanishi ${ }^{18}$, são semelhantes ao de Akkurt et al. $\frac{12}{}$, verificando que a melhora do condicionamento físico através da prática de exercícios físicos no meio por exemplo, pode contribuir para a redução de comorbidades, promoção de saúde e qualidade de vida dos pacientes com TRM.

O uso da realidade virtual apresentou-se como um recurso promissor, onde foram encontrados resultados significativos no tratamento de pacientes com TRM. Estudos como o de Leão et al. ${ }^{13}$ e Maresca et al. ${ }^{14}$ afirmam que o tratamento com RV foi benéfico para o ganho da função motora, equilíbrio, coordenação e autonomia funcional em indivíduos jovens. Além destes benefícios, quando a RM foi aplicada em adultos, houve redução de sintomas de ansiedade e depressivos melhorando a QV dos pacientes com TRM.
A RV é um recurso que pode ser utilizado para estimular os movimentos durante os jogos interativos, às tecnologias presentes neste recurso criam informações sensoriais artificiais em um ambiente virtual, simulando o mundo real, incentivando a atividade física e ganho de equilíbrio Darekar et al. ${ }^{19}$. Contudo, a literatura apresenta poucos resultados relacionados à $\mathrm{RV}$ como um método de tratamento em pacientes com TRM, dificultando a comprovação e eficácia deste método ${ }^{20}$. Assim, ressalta-se a importância de mais pesquisas que abordem essa questão.

Além das limitações funcionais, outras variáveis podem comprometer o bem-estar físico e mental dos indivíduos acometidos por TRM, como dificuldade de socialização, inatividade sexual, lesão por pressão e dor, que podem interferir diretamente em sua QV. De acordo com Mota; Ribeiro (2016), em estudo transversal composto de 118 pacientes com idade média de 34,7 anos, observou-se alta prevalência de lesão por pressão em pessoas com TRM, sendo a avaliação da qualidade de vida elucidada por uma insatisfação significativa referente aos aspectos físicos. Outro estudo com abordagem metodológica semelhante, identificou a dor como um fator que diminui a QV dos pacientes estudados ${ }^{11}$.

Para o alívio da dor e recuperação funcional, a fisioterapia atua com utilização de diversas técnicas e recursos, dentre eles a eletroterapia e cinesioterapia. O uso da eletroterapia reduz a espasticidade e a dor mostraram melhora significativa no quadro do paciente, onde é possível uma reintegração social, diminuição de sintomas depressivos e retorno ao trabaIho que melhoram a QV15,16. 
A fisioterapia no trato de pacientes com LM tem se mostrado cada vez mais importante, tanto no auxílio na diminuição de dores quanto nas orientações e intervenções da prevenção ou redução das complicações antes e pós lesão, utilizando tratamento multidisciplinar na melhora da qualidade de vida, na sua reabilitação e readaptação do paciente a vida social21.

De acordo com a literatura o apoio familiar, orientações de uma equipe multiprofissional e a reabilitação focada na capacidade motora ajudam na promoção do bem-estar físico e mental assim como na reeducação sexual, social, emocional que geram impactos positivos na qualidade de vida desses pacientes ${ }^{1,22}$.

Considerando os estudos apresentados verificou-se que houve diversas variáveis abordadas com relação aos pacientes com TRM, sendo a fisioterapia de suma importância para a QV desses pacientes. No entanto, algumas limitações foram identificadas na realização desta pesquisa, como os tipos de estudos encontrados e suas amostras pequenas. Diante disso, sugere-se mais estudos sobre a temática abordada.

\section{Conclusão}

Foi verificado que diversas abordagens podem contribuir para a melhor qualidade de vida dos pacientes com TRM, sendo de suma importância o acompanhamento profissional especializado para o bem-estar físico e psicológico desses pacientes. Contudo, sugere-se mais pesquisas sobre a temática estuda para oferecer meios de tratamentos que aumentem a QV dos pacientes com TRM.

\section{Contribuições dos autores}

Silva FVM contribuiu com o delineamento, estruturação, escrita da redação e revisão crítica do manuscrito. Silva ANJ, Castro DMP, Ribeiro RP, Sales TO contribuiram com busca de artigos, escrita e redação manuscrito. Nunes PPB contribuiu com o delineamento, estruturação, escrita da redação e revisão crítica do manuscrito.

\section{Conflitos de interesses}

Nenhum conflito financeiro, legal ou político envolvendo terceiros (governo, empresas e fundações privadas, etc.) foi declarado para nenhum aspecto do trabalho submetido (incluindo, mas não se limitando a subvenções e financiamentos, participação em conselho consultivo, desenho de estudo, preparação de manuscrito, análise estatística, etc.).

\section{Referências}

1. Torrecilha LA, Costa BT, Lima FB, Santos SMS, Souza RB. O perfil da sexualidade em homens com lesão medular. Fisioter Mov. 2014;27(1):39-48. doi: 10.1590/0103-5150.027.001.AO04

2. Souza FBV, Castro PCG, Ayres DVM, Moreira MCS, Battistella LR. Benefits of robotic-assisted gait in spinal cord injury: a systematic review. Acta Fisiátrica. 2013;20(3):142-6. doi: 10.5935/0104$\underline{7795.20130023}$

3. Frison VB, Teixeira GO, Oliveira TF, Resende TL, Alexandre Netto C. Estudo do perfil do trauma raquimedular em Porto Alegre. Fisioter Pesqui. 2013;20(2):165-71.doi: 10.1590/S180929502013000200011

4. Citero EM, Mederdrut EM, Fontes SV. Efeitos da Fisioterapia com Trampolim no Traumatismo Raquimedular: Rev Neurocienc. 2011;20(2):222-32. doi: 10.34024/rnc.2012.v20.8287

5. Tse CM, Chisholm AE, Lam T, Eng JJ. A systematic review of the effectiveness of task-specific rehabilitation interventions for improving independent sitting and standing function in spinal cord injury. J Spinal Cord Med. 2018;41(3):254-66. doi: $\underline{10.1080 / 10790268.2017 .1350340}$

6. Pereira ÉF, Teixeira CS, Santos A. Qualidade de vida: abordagens, conceitos e avaliação. Rev Bras Educ Fís Esporte. 2012;26(2):241-50. doi: 10.1590/S1807-55092012000200007

7. Pérez-Parra JE, Henao-Lema CP. Relación entre complicaciones clínicas y discapacidad en población colombiana con lesion medular: Resultados desde el WHO-DAS II. Aquichan. 2013;13(2):173-85. doi: 10.5294/aqui.2013.13.2.3

8. Souza MT, Silva MD, Carvalho R. Integrative review: what is it? How to do it? Einstein. 2010;8(1):102-6. doi: 10.1590/s167945082010 rw1134

9. Feniman SP, Cardoso JR, Villegas I, Bela LFD, Santos SM, Lavado EL. Development and validation of a quality of life questionnaire for individuals with spinal cord injury. Acta Fisiátrica. 2016;23(4):172-9. doi: 10.5935/0104-7795.20160033 
10. Mota D, Ribeiro MB. Quality of life in patients with spinal cord injury and pressure ulcers. Enferm Glob. 2016;15(42):13-21. doi: 10.6018/eglobal.15.2.210971

11. Richardson EJ, Brooks LG, Richards JS, Bombardier $\mathrm{CH}_{\text {, }}$ Barber J, Tate D et al. Changes in pain and quality of life in depressed individuals with spinal cord injury: does type of pain matter? J Spinal Cord Med. 2016;39(5):535-43. doi: 10.1080/10790268.2016.1151145

12. Akkurt H, Karapolat HU, Kirazli Y, Kose T. The effects of upper extremity aerobic exercise in patients with spinal cord injury: A randomized controlled study. Eur J Phys Rehabil Med. 2017;53(2):219-27. doi: 10.23736/S1973-9087.16.03804-1

13. Leão CD, Barros GM, Santos MCS, Oliveita LS. Impacto da realidade virtual no equilíbrio e na qualidade de vida em indivíduos com lesão medular. Rev Bras Ciênc Mov. 2017;25(1):6978. doi: $10.18511 / \mathrm{rbcm} . \mathrm{v} 25 \mathrm{i1} .6301$

14. Maresca G, Maggio MG, Buda A, La Rosa G, Manuli A, Bramanti $P$ et al. A novel use of virtual reality in the treatment of cognitive and motor deficit in spinal cord injury A case report. Medicine. 2018;97(50):e13559.. doi: 10.1097/MD.0000000000013559

15. Sivaramakrishnan A, Solomon JM, Manikandan N. Comparison of transcutaneous electrical nerve stimulation (TENS) and functional electrical stimulation (FES) for spasticity in spinal cord injury - A pilot randomized cross-over trial. J Spinal Cord Med. 2018;41(4):397-406. doi: 10.1080/10790268.2017.1390930

16. Calliga MCNS, Porto LA. Which people with traumatic paraplegia return to work? Cienc Saude Coletiva. 2019;24(6):234150. doi: $10.1590 / 1413-81232018246.15682017$
17. Almeida PA, Barbosa VRC, Dias MC, Neiva CM. Efeitos da prática da natação adaptada sobre o perfil bioquímico e o estado de condicionamento físico de indivíduos com lesão medular. Medicina. 2011;44(4):377-88. doi: 10.11606/issn.2176-7262. v44i4p377-388

18. Kawanishi CY, Greguol M. Avaliação da Autonomia Funcional de Adultos com Lesão Medular. Rev Ter Ocup da Univ São Paulo. 2014;25(2):159-66. doi: 10.11606/issn.2238-6149.v25i2p159-166

19. Darekar A, McFadyen BJ, Lamontagne A, Fung J. Efficacy of virtual reality-based intervention on balance and mobility disorders post-stroke: A scoping review. J Neuroeng Rehabil. 2015;12(1):46. doi: 10.1186/s12984-015-0035-3

20. Robinson J, Dixon J, Macsween A, van Schaik P, Martin D. The effects of exergaming on balance, gait, technology acceptance and flow experience in people with multiple sclerosis: A randomized controlled trial. BMC Sports Sci Med Rehabil. 2015;7:8. doi: 10.1186/s13102-015-0001-1

21. Rodrigues AV, Vidal WAS, Lemes JA, Gôngora CS, Neves TC, Smaili SM et al. Study on the characteristics of pain in patients with spinal cord injury. Acta Fisiátrica. 2012;19(3):171-7. doi: $\underline{10.5935 / 0104-7795.20120027}$

22. França ISX, Coura AS, Sousa FS, Almeida PC, Pagliuca LMF. Qualidade de vida em pacientes com lesão medular. Rev Gaúcha Enferm. 2013;34(1):155-63. doi: 10.1590/S198314472013000100020 